\title{
Development of a rapid and sensitive immunochromatographic strip based on EuNPs-ES fluorescent probe for the detection of early Trichinella spiralis-specific lgG antibody in pigs
}

Xinyu Wang ${ }^{1 \dagger}$, Bin Tang ${ }^{1 \dagger}$, Ying Zhao ${ }^{2 \dagger}$, Jing Ding ${ }^{1}$, Nan Wang ${ }^{4}$, Yan Liu ${ }^{1}$, Zijian Dong ${ }^{1}$, Xiangdong Sun ${ }^{5}$, Quangang $\mathrm{Xu}^{5}$, Mingyuan Liu ${ }^{1,3^{*}}$ and Xiaolei Liu ${ }^{1 *}$

\begin{abstract}
Trichinellosis, which is caused by nematodes of the genus Trichinella, is one of the most important zoonotic parasite diseases in the world. A rapid and sensitive immunochromatographic strip (ICS) based on Eu (III) nanoparticles (EuNPs) was developed for the detection of Trichinella spiralis (T. spiralis) infection in pigs. T. spiralis muscle larvae excretory secretory or preadult worm excretory secretory (ML-ES or PAW-ES) antigens were conjugated with EuNPs probes to capture T. spiralis-specific antibodies in pig sera, after which the complex bound to mouse anti-pig IgG deposited on the test line (T-line), producing a fluorescent signal. In the pigs infected with 100, 1000 and 10000 ML, seroconversion was first detectable for the EuNPs-ML-ES ICS at 30, 25 and 21 days post-infection (dpi) and for the EuNPs-PAW-ES ICS at 25, 21 and $17 \mathrm{dpi}$. These results show that EuNPs-PAW-ES ICS detects anti-Trichinella IgG in pigs 4-5 days earlier that test using ML-ES antigens. Our ICS have no cross reaction with other parasite infection sera. Furthermore, the detection process could be completed in $10 \mathrm{~min}$. This study indicated that our ICS can be used for the detection of the circulating antibodies in early T. spiralis infection and provide a novel method for on-site detection of T. spiralis infection in pigs.
\end{abstract}

Keywords: Trichinella spiralis, Excretory-secretory, Eu (III) nanoparticles, Immunochromatographic strip, Serodiagnosis

\section{Introduction}

Trichinellosis is a parasitic zoonotic disease caused by nematodes of the genus Trichinella, which have a global geographical distribution and affects a wide variety of more than 150 carnivorous and omnivorous animals [13]. Most of human infections are caused by Trichinella

\footnotetext{
*Correspondence: liumy36@163.com; liuxlei@163.com

${ }^{+}$Xinyu Wang, Bin Tang and Ying Zhao contributed equally to the work ${ }^{1}$ Key Laboratory of Zoonosis Research, Ministry of Education,

Institute of Zoonosis, College of Veterinary Medicine, Jilin University, Changchun 130062, China

Full list of author information is available at the end of the article
}

spiralis (T. spiralis) by consuming raw or uncooked meat containing infective muscle larvae (ML), and humans usually present with clinical symptoms [3]. According to a recent report by the International Commission on Trichinellosis (ICT), the number of humans infected with Trichinella spp. was 65818 from 1986 to 2009 [4]. Meanwhile, 38794 cases of T. spiralis infection were reported in China from 1964 to 2011, of which 336 led to death [5-7]. From 2000 to 2015, 2251 people were diagnosed as trichinellosis in Yunnan Province, China [8]. Therefore, China has become a region greatly affected by trichinellosis in recent decades. In view of the above situation,

(c) The Author(s) 2021. This article is licensed under a Creative Commons Attribution 4.0 International License, which permits use, sharing, adaptation, distribution and reproduction in any medium or format, as long as you give appropriate credit to the original author(s) and the source, provide a link to the Creative Commons licence, and indicate if changes were made. The images or other third party material in this article are included in the article's Creative Commons licence, unless indicated otherwise in a credit line to the material. If material is not included in the article's Creative Commons licence and your intended use is not permitted by statutory regulation or exceeds the permitted use, you will need to obtain permission directly from the copyright holder. To view a copy of this licence, visit http://creativeco mmons.org/licenses/by/4.0/. The Creative Commons Public Domain Dedication waiver (http://creativecommons.org/publicdomain/ zero/1.0/) applies to the data made available in this article, unless otherwise stated in a credit line to the data. 
China has spent 2.2 billion CNY each year on preventing and detecting trichinellosis [5]. Moreover, pigs are the most common vector for transmitting trichinellosis, which is primarily caused by $T$. spiralis infection in China [9]. Therefore, to ensure food safety and maintain the economic stability of the breeding pig industry, there is an urgent need to develop a method of on-site detection of $T$. spiralis in pigs.

After the host ingests the meat containing T. spiralis, the infective larvae are released in the muscle tissue of the ingested meat and migrate into the small intestine. Then, they shed four molts and develop into adult worms (AW) within 30-36 h post-infection. Four days later, female adult worms release newborn larvae (NBL) which arrive the host muscle tissue through the circulation of blood and lymph fluid and parasitize in muscle cell for years. Muscle larvae excretory secretory (MLES) antigens are produced by T. spiralis ML, and ML-ES antigens are commonly used in serological methods for the diagnosis of Trichinella infections in animals and humans [10-12]. However, ML-ES antigens are phasespecific and can cause false negatives in the $3^{\text {rd }}-4^{\text {th }}$ weeks post-infection [13]. The false negatives are due to the lag time of the immune response following the ingestion of infective larvae. Therefore, the ELISA or immunochromatographic strip (ICS) methods based on ML-ES antigens cannot be recommended for the detection of early T. spiralis infections. Previous study reported that adult worm excretory secretory (AW-ES) can be identified by the anti-Trichinella antibodies in early $T$. spiralis infection $[14,15]$. Meanwhile, preadult worm (PAW) obtained at $6 \mathrm{~h}$ post-infection makes early contact with the host immune system compared with AW $[16,17]$. Therefore, ML-ES and PAW-ES antigens were applied and evaluated for the detection of T. spiralis infection by ICS in this study.

The diagnosis of Trichinella infection utilizes direct and indirect methods, and the gold standard method is the artificial digestion method that is recommended by the World Organization for Animal Health (OIE). The ELISA detection method based on ML-ES antigens is a widely accepted serological method for monitoring Trichinella infection and investigating its epidemiology $[18,19]$. However, the above described methods have the disadvantage of long detection time, laborious and expensive equipment. With the development of immunochromatography technology, ICS methods that utilize gold nanoparticles, up-converting phosphor nanoparticles, magnetic particles and quantum dots have been widely used in testing for pregnancy and chemical residues [20-22]. Moreover, colloidal gold nanoparticles as labels on ICS have been used to detect $T$. spiralis infection [23, 24]. Recently, EuNPs, which are time-resolved fluorescent microspheres with the advantages of a large Stokes shift, a narrow emission spectrum and a long quenching time, have begun to replace colloidal gold as the labels used in mainstream ICS methods for clinical disease detection, such as hepatitis B virus and porcine epidemic diarrhea virus $[25,26]$. For investigating the seroconversion of infected pigs, a novel EuNPs-ICS detection method based on ML-ES antigens and PAW-ES antigens was developed for $T$. spiralis infection.

In this study, an EuNPs-ES fluorescent probe conjugated with ML-ES and PAW-ES antigens were established for detecting T. spiralis-specific IgG antibodies in pigs by ICS, respectively. The sensitivity and specificity of two kinds of ICS were investigated, which will help promoting the on-site diagnosis of Trichinella infection. To date, there has no reports on detecting T. spiralis infection by fluorescent probe-based ICS.

\section{Materials and methods \\ Reagents and instruments}

Bovine serum albumin (BSA) and Tween-20 were obtained from Solarbio (Beijing, China). EDC was obtained from TCI (Shanghai, China). $\mathrm{COOH}$-modified europium nanoparticles (EuNPs) were obtained from Thermo (USA). Mouse anti-pig monoclonal IgG antibodies and rabbit anti-goat IgG antibodies were obtained from Beijing Biolab Technology (Beijing, China). Goat anti-rabbit IgG was obtained from Cell Signal Technology (USA). NC membranes (Millipore 135) and Ultra-15 $3 \mathrm{kDa}$ centrifugal filters were obtained from Millipore (USA). The sample pads, absorbent paper and plastic backing were obtained from Jinbiao Biotech (Shanghai, China). BCA kits were obtained from Beyotime Biotechnology (Shanghai, China). The ES commercial ELISA kit was obtained from Qiagen (Germany).

XYZ3060 dispenser was obtained from Biodot (USA). A TRF fluorescence quantitative analyzer was obtained from Weice Biotech (Nanjing, China). A UV lamp was obtained from Shenzhen Feike Technology (Shenzhen, China).

\section{Preparation of ES antigens}

The collection of ML-ES antigens followed the previous methods and procedures [27, 28]. A total of 10 specific pathogen-free (SPF) SD rats were orally inoculated with 3500 T. spiralis ML (T1, ISS534) and were euthanized at $35 \mathrm{dpi}$, and tissues were used for the recovery of ML by the artificial digestion method. After washing three times with $0.9 \%$ saline solution, the ML were cultured in serum-free RPMI-1640 medium containing antibiotics (90 U/mL penicillin and $90 \mu \mathrm{g} / \mathrm{mL}$ streptomycin) at $37^{\circ} \mathrm{C}$ for $18 \mathrm{~h}$ in $5 \% \mathrm{CO}_{2}$. After separation by centrifugation at $1000 \times g$, the ML culture supernatant was concentrated 
by Ultra-15 $3 \mathrm{kDa}$ centrifugal filters and the protein concentration was measured by BCA kits.

The collection of PAW-ES antigens followed the previous methods and procedures [14]. After infection with 10000 T. spiralis ML, the SD rats were euthanized at $6 \mathrm{~h}$ after infection. The entire small intestine was removed from the abdominal cavity and soaked in $0.9 \%$ saline solution at $37^{\circ} \mathrm{C}$ for $2 \mathrm{~h}$ (with $180 \mathrm{U} / \mathrm{mL}$ penicillin and $180 \mu \mathrm{g} / \mathrm{mL}$ streptomycin). The culture and collection of PAW-ES antigens were performed as ML-ES antigens.

Finally, ML-ES antigens and PAW-ES antigens were analyzed by SDS-PAGE (Additional file 1).

\section{Pigs and serum samples}

The experiment of this part has been done by our laboratory before [27]. Fifteen Large White pigs were divided into three groups (five pigs per group) and inoculated with 100, 1000 and 10000 T. spiralis ML (T1, ISS534). Serum samples were collected from pigs at $0,7,9,11,13,15,17,19,21,25,30,35$, $45,60,90$ and 120 dpi. Finally, all pigs were sacrificed to calculate larvae per gram of muscle (lpg) at $120 \mathrm{dpi}$. These results were showed in supplement material, which indicated all pigs were successfully infected with $T$. spiralis ML (Additional file 4).

One hundred serum samples were collected from 25 Large White pigs infected with 400 T. spiralis ML at 25, 30, 35, 45 dpi. Another 170 serum samples were collected from 170 parasite-free Large White pigs as negative controls to calculate the cut-off value. A total of 38 serum samples were collected from pigs infected with Clonorchis sinensis (2), Cryptosporidium parvum (2), Taenia sodium (2), Toxoplasma gondii (2), Ascaris suum (15), Trichuris suis (5) and Metastrongylus elongatus (10). All pigs were healthy according to the Chinese Laboratory General Requirements for Animal Experiments. Before the experiment, all pigs underwent a week-long healthy observation and were examined for parasite eggs in feces and blood by the flotation and sedimentation method. Furthermore, all pigs were fed basic diet without adding antibiotics and were kept under standard pig houses in our laboratory under the care of a professional breeder.

\section{Preparation of fluorescent probe}

EuNPs were conjugated with ES antigens as follows [29]: firstly, $10 \mu \mathrm{L}$ of EuNPs were centrifuged at $14000 \times g$ for $15 \mathrm{~min}$ to remove glycerol and phosphate, and then $10 \mu \mathrm{L}$ of $1 \mathrm{mg} / \mathrm{mL}$ EDC, $10 \mu \mathrm{L}$ of $1 \mathrm{mg} / \mathrm{mL}$ NHS, $100 \mu \mathrm{L}$ of MES and EuNPs were mixed and stirred for $45 \mathrm{~min}$ to activate the EuNPs completely at room temperature. After removing the unbound EDC and NHS by centrifugation at $14000 \times g$ for $15 \mathrm{~min}, 20 \mu \mathrm{g}$ the ML-ES and $20 \mu \mathrm{g}$ PAW-ES antigens were mixed into the solution, and incubated for $3 \mathrm{~h}$. Then, $200 \mu \mathrm{L}$ of $5 \%$ BSA was added to the mixtures to block the unreacted active sites overnight at $4{ }^{\circ} \mathrm{C}$. Finally, the EuNPs-ML-ES and EuNPsPAW-ES fluorescent probes were resuspended in $200 \mu \mathrm{L}$ of preservation buffer $(50 \mathrm{~mm} / \mathrm{L}$ PBS containing $1 \%$ BSA and $1 \%$ ProClin). The EuNPs-goat anti-rabbit IgG fluorescent probe was also prepared by the same procedure described above.

To identify the change of fluorescent probe after coupling, the morphology of the fluorescent probe was observed by transmission electron microscopy (TEM) and the absorption peak of the fluorescent probe was detected by fluorescent microplate reader.

\section{Preparation of the ICS}

EuNPs-conjugated ML-ES or PAW-ES antigens were designed as probes to capture anti- $T$. spiralis antibodies, and conjugated goat anti-rabbit IgG antibodies were used as an indicator probe. Mouse anti-pig monoclonal IgG and rabbit anti-goat IgG were immobilized on the $\mathrm{NC}$ membrane as the test line (T-line) and control line (C-line), respectively. Using the XYZ3060 dispenser at a rate of $0.8 \mu \mathrm{L} / \mathrm{cm}$, mouse anti-pig monoclonal IgG $(1 \mathrm{mg} /$ $\mathrm{mL})$ and rabbit anti-goat IgG $(1 \mathrm{mg} / \mathrm{mL})$ were applied to the $\mathrm{NC}$ membrane as the $\mathrm{T}$-line and $\mathrm{C}$-line, respectively. Finally, NC membranes absorbed paper and sample pad were assembled into strips ( $3.8 \mathrm{~mm}$-wide).

For detection, $100 \mu \mathrm{L}$ the running buffer $(0.9 \% \mathrm{NaCl}$, 1.5\% BSA, 0.05\% Tween-20), $1 \mu \mathrm{L}$ serum sample, $1 \mu \mathrm{L}$ EuNPs-ES and $0.5 \mu \mathrm{L}$ EuNPs-goat anti-rabbit IgG were mixed in a bioclean tube. Then, after the sample was added into the ICS, the ICS was placed into a $37^{\circ} \mathrm{C}$ incubator for $10 \mathrm{~min}$. The fluorescent signal of the T-line was observed under a UV lamp at $365 \mathrm{~nm}$ wavelength and was analyzed by a TRF reader.

\section{The cut-off value for the ICS and Qiagen ELISA}

A total of 170 serum samples from 170 parasite-free pigs were detected by ICS, and the result of ICS were analyzed by a TRF reader. The cut-off value was calculated by $\bar{x} \pm 2$ SD of the T-line fluorescence values of the 170 serum samples. A T-line values for a sample below the cut-off value was judged as negative, and a value above or equal to the cut-off value was judged as positive. The cut off value of Qiagen ELISA follow the manufacturer's instructions: $\mathrm{S} / P$ values $=\left(\mathrm{OD}_{\text {Sample }}-\mathrm{OD}_{\text {Negative Control }}\right) /$ $\left(\mathrm{OD}_{\text {Positive Control }}-\mathrm{OD}_{\text {Negative Control }}\right)$.

\section{Cross-reactivity with other parasites}

To evaluate the specificity of ICS, a total of 38 serum samples from pigs infected with Clonorchis sinensis, Cryptosporidium parvum, Taenia sodium, Toxoplasma gondii, Ascaris suum, Trichuris suis and Metastrongylus 
elongatus were detected by the EuNPs-ML-ES ICS and EuNPs-PAW-ES ICS.

\section{Seroconversion of infected pigs detected by ICS and Qiagen ELISA}

Serum samples from pigs infected with 100, 1000 and 10000 ML (five animals per group) at 0, 7, 9, 11, 13, 15, 17, 19, 21, 25, 30, 35, 45, 60, 90 and 120 dpi were detected by EuNPs-ML-ES ICS, EuNPs-PAW-ES ICS and Qiagen ELISA. The test results for the middle- and high-dose ELISA groups have been published previously, and the results for the low-dose group are shown in Additional file 2 [30].

A standard indirect ELISA protocol was performed by Qiagen ELISA kit. Briefly, $90 \mu \mathrm{L}$ of sample diluent and $10 \mu \mathrm{L}$ prediluted serum were added into each sample well, then wells were incubated for $60 \mathrm{~min}$ at room temperature $\left(18-25^{\circ} \mathrm{C}\right)$. After removing solution by aspiration, wells were rinsed each well of Wash Buffer. $100 \mu \mathrm{L}$ ready-to-use Conjugation was added into each well and incubated for $30 \mathrm{~min}$ at room temperature. After removing solution and wells were rinsed again. $100 \mu \mathrm{L}$ TMB Substrate Solution was added into each well and incubated for $10 \mathrm{~min}$ at room temperature. Stop the reaction by adding $100 \mu \mathrm{L}$ Stop Solution per well, and optical density (OD) values was measured by plate reader at $450 \mathrm{~nm}$.

\section{The sensitivity and specificity of EuNPs-ML-ES ICS and EuNPs-PAW-ES ICS}

To investigate the sensitivity and specificity of ICS, 100 serum samples were collected from 25 Large White pigs infected with $400 \mathrm{~T}$. spiralis ML at 25, 30, 35, $45 \mathrm{dpi}$, at the end of experiment, all pigs were confirmed to be infected with $T$. spiralis ML by artificial digestion method. 170 serum samples collected from 170 parasite-free pigs as negative, which also confirm by artificial digestion method. These serum samples were detected by EuNPs-ML-ES ICS and EuNPs-PAW-ES ICS.

\section{Clinical application of ICS}

To validate the method in the field in an endemic area where Trichinella circulates among the backyard pigs. We collected 1032 pork and 1032 serum samples from slaughterhouses and rural areas in Inner Mongolia, Sichuan and Guangxi provinces in China where T. spiralis infection were suspected. All pork samples were detected by artificial digestion method, and serum sample were detected by the two ICS.

\section{Statistical analysis}

After the results of ICS were analyzed by fluorescent reader, the T-line fluorescence values were imported into GraphPad Prism8 software. SPSS 19 was used for statistical analysis, Chi-square tests were performed on ICS with standard artificial digestion.

\section{Results \\ The process of immunochromatography}

The ICS used for detecting anti-T. spiralis antibodies is based on a classic indirect method. As shown in Figure $1 \mathrm{~A}$, when a positive serum sample was detected, the EuNPs-ES probe captured the anti-T. spiralis antibodies. Then, under the traction of the absorption pad, the mixture migrated across the $\mathrm{NC}$ membrane and reacted with the mouse anti-pig monoclonal IgG antibodies on the T-line. Meanwhile, the EuNPs-goat anti-rabbit IgG antibodies probe reacted with the rabbit anti-goat IgG antibodies on the $\mathrm{C}$-line. When the negative sample was detected, only the EuNPs-goat anti-rabbit IgG probe was captured on the C-line (Figure 1B).

\section{The diagnosis of EuNPs-ICS}

The EuNPs probe was imaged by TEM (Figure 2A). It is clear that the two probes have a uniform circular shape and almost the same diameter. The maximal emission peak appeared in $625 \mathrm{~nm}$ and there was no difference between EuNPs and EuNPs-ES probe (Figure 2B). When serum samples were detected by the ICS, a strong fluorescence signal appeared on the T-line for the positive serum sample, but no fluorescence signal was found on the T-line for the negative serum sample. In addition, regardless of whether positive or negative serum sample was detected, the C-line showed a certain degree of fluorescence, which indicated that the ICS could distinguish negative and positive serum sample (Figures $2 \mathrm{C}$ and $\mathrm{D}$ ).

\section{The cut-off value of the ICS}

Normal 170 serum samples from parasite-free pigs were detected by the ICS. Based on the means $\pm 2 S D$ value, the cut-off value for the EuNPs-ML-ES ICS and the EuNPsPAW-ES ICS were 115.71 and 112.97, respectively. Interestingly, the cut off value of EuNPs-ML-ES ICS is slightly high than EuNPs-PAW-ES ICS.

Moreover, the T-line fluorescence values for 170 serum samples were imported into GraphPad Prism8 software, and we found that the data conformed to a normal distribution (Figure 5).

\section{Cross-reactivity with other parasites serum samples}

The T-line fluorescence values for serum samples from other parasites were below the cut-off value, and no positive bands appeared in ICS for other parasites serum samples, which indicated that the EuNPs-ICS has no cross-reactivity with other parasites serum samples (Figure 3). 


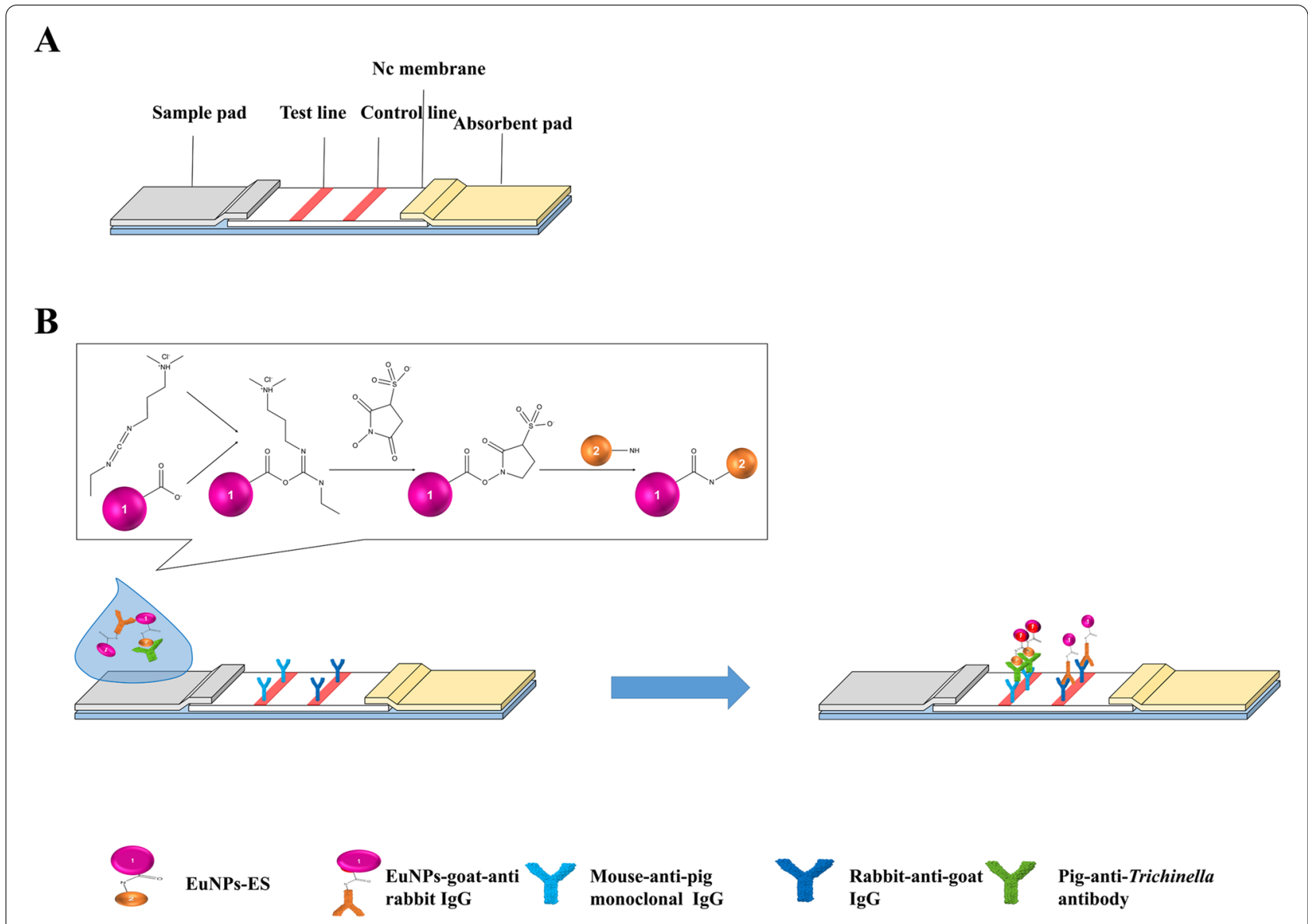

Figure 1 The schematic of EuNPs-ICS. A The composition of immunochromatographic strips. B The process of immunochromatographic strips.

\section{Seroconversion of infected pigs detected by ICS and Qiagen ELISA}

As shown in Figure 4, the fluorescence intensity of the $\mathrm{T}$-line increased as the number of dpi or ML infection doses increased. Based on the cut-off value, in the pigs infected with 100, 1000 and $10000 \mathrm{ML}$, seroconversion was first detectable by the EuNPs-ML-ES ICS at 30, 25 and 21 dpi and by the EuNPs-PAW-ES ICS at 25, 21 and $17 \mathrm{dpi}$, respectively (Figure 4). In the pigs infected with 100, 1000 and $10000 \mathrm{ML}$, $100 \%$ antibody positivity was detected by the EuNPs-MLES ICS at 45, 30 and 25 dpi and by the EuNPs-PAW-ES ICS at 35, 25 and 19 dpi (Table 1). When serum samples were detected by Qiagen ELISA, in pigs infected with 100, 1000 and $10000 \mathrm{ML}$, seroconversion was first detectable at 30, 25, and $21 \mathrm{dpi}$, and $100 \%$ antibody positivity was detected at 45 , 30 and 25 dpi (Table 1). These results indicated seroconversion of infection pigs was detected by EuNPs-PAW ICS earlier than EuNPs-ML-ES ICS and Qiagen ELISA.

\section{The sensitivity and specificity of EuNPs-ML-ES ICS and EuNPs-PAW-ES ICS}

The sensitivity of the EuNPs-ML-ES ICS and EuNPs-PAWES ICS was $85 \%$ and $97 \%$, respectively. The specificity of the EuNPs-ML-ES ICS and EuNPs-PAW-ES ICS was $96.47 \%$ and $95.29 \%$, respectively (Figure 5 ). The Chi-square test of golden standard artificial digestion and EuNPs-PAWES ICS gave a $P$ value of $0.227\left(\mathrm{McNemar} \chi^{2}, P>0.05\right)$, and Kappa values of 0.917 . The Chi-square test of golden standard artificial digestion and EuNPs-ML-ES ICS gave a $P$ value of 0.078 (McNemar $\chi^{2}, P>0.05$ ), and Kappa values of 0.83 (Table 2). Compared with EuNPs-ML-ES ICS, EuNPs-PAWES ICS could distinguish precisely positive and negative sera and showed a strong agreement with the artificial digestion methods.

\section{Clinical application of ICS}

For investigating the performance of clinical application, artificial digestion and ICS method were evaluated by detection of clinical samples. As shown in Table 3, 
A

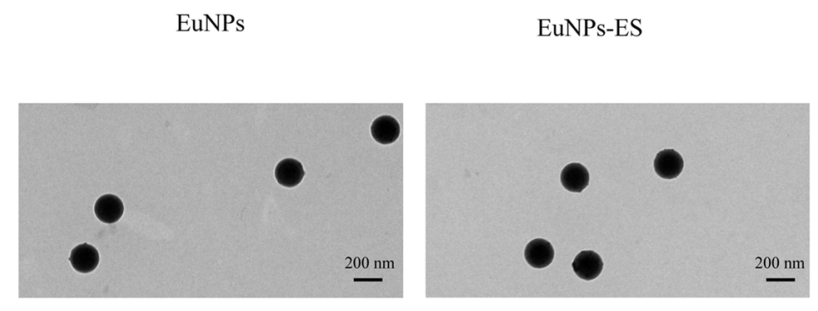

C

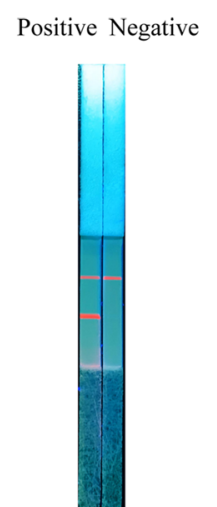

B

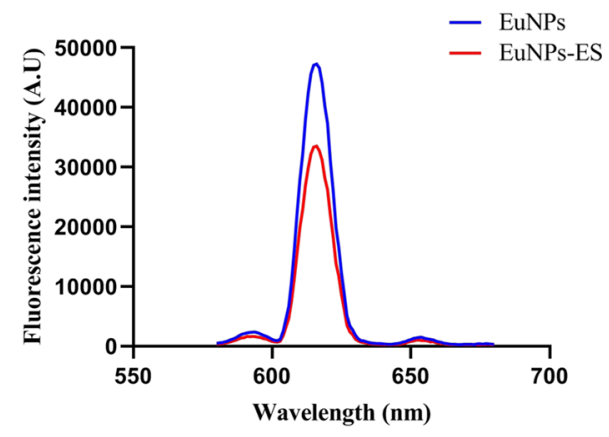

D

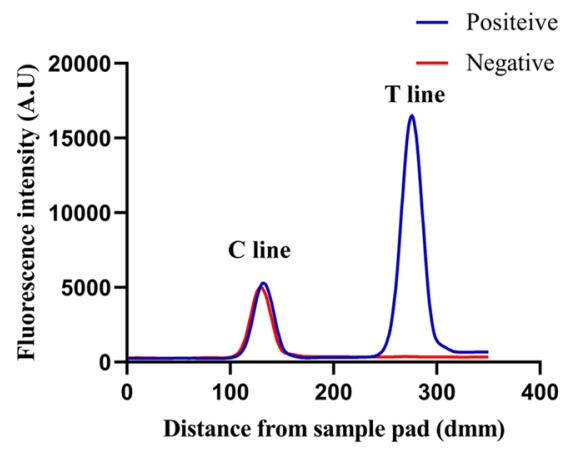

Figure 2 The diagnosis of EuNPs-ICS. A The image of EuNPs or EUNPs-ES probe by TEM. B The maximal emission peak of EuNPs or EuNPs-ES probe. C The image of positive and negative serum samples detected by ICS under UV lamp. D Fluorescence peak heights readout curve of ICS for detecting positive and negative serum samples.

In total of 1032 serum samples and 1032 pork samples, three serum samples were detected as antibody positive by ICS, one pork sample was detected as positive by artificial digestion (Additional file 3, Table 3).

\section{Discussion}

Established a rapid and sensitive detection method for T. spiralis infection is a priority topic. Due to the advantages of a large stokes shift, a narrow emission spectrum and a long quenching time, the EuNPs-ICS shows high sensitivity and specificity compared to the colloidal gold ICS and is widely used in disease diagnosis, food safety, public health and environmental protection [31, 32]. However, EuNPs-ICS has not been applied in detection of T. spiralis infection. In this study, a novel and rapid EuNPs-ICS was developed for investigating seroconversion of infected pigs, and the specificity and sensitivity of ICS were evaluated.

ES antigens are secreted by the cuticle surface and stichosome of T. spiralis [33]. Tyvelose, the component of the ES antigens, is also found in Gram-negative bacterial lipopolysaccharides and Ascaris eggs, so the serological method established by using the ES antigens has cross-reactivity with sera from infection with other parasites [34]. To improve the specificity of ICS, anti-pig polyclonal IgG antibodies were replaced with mouse anti-pig monoclonal IgG antibodies as the T-line capture antibodies. To enhance the blocking effect, different concentrations of BSA was added into coupling system and loading buffer system. Our results indicate that our ICS have high specificity and have no cross reaction with sera from infection with other parasites.

ELISA method based on ML-ES antigens is commonly used in diagnosis of T. spiralis infection. But ML-ES antigens are phase-specific, the false negative results were often obtained by ELISA method on detection of early T. spiralis infection [13]. In this study, PAW-ES and ML-ES antigens were applied in EuNPs-ICS to investigate the seroconversion of infection pigs compared with Qiagen ELISA. The limit detection of EuNPs-ML-ES ICS was consistent with Qiagen ELISA, whereas the limit detection of EuNPs-PAW-ES ICS was lower than Qiagen ELISA, suggesting that PAW-ES antigens are more sensitive to antibodies produced in early $T$. spiralis infection. Moreover, our previous study showed that T. spiralis infection first be detectable at $17 \mathrm{dpi}$ by standard artificial digestion [27]. EuNPs-PAW-ES ICS gives a high 
A
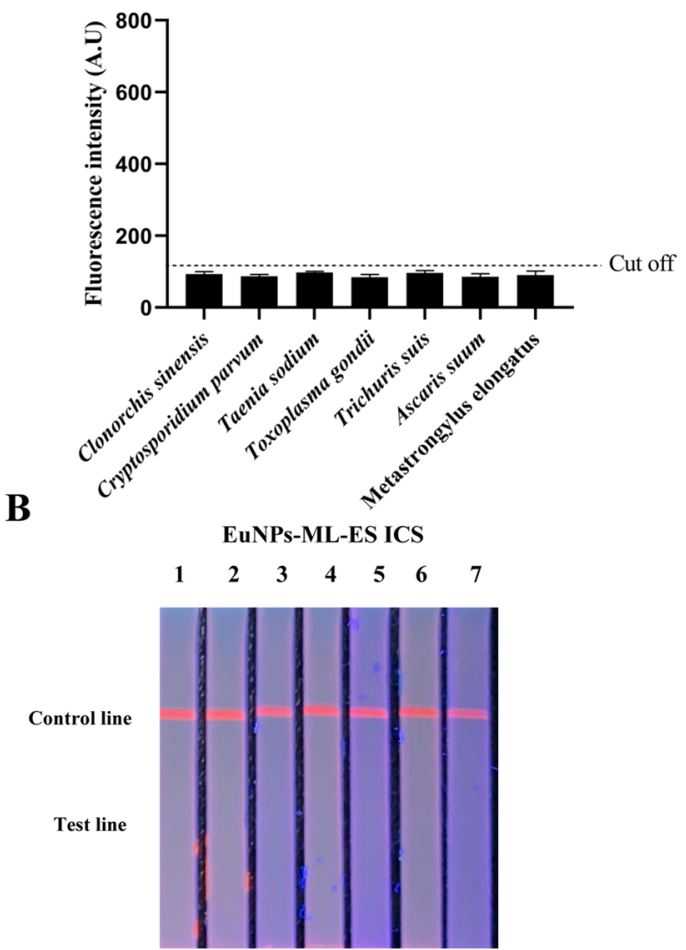

$\mathbf{C}$

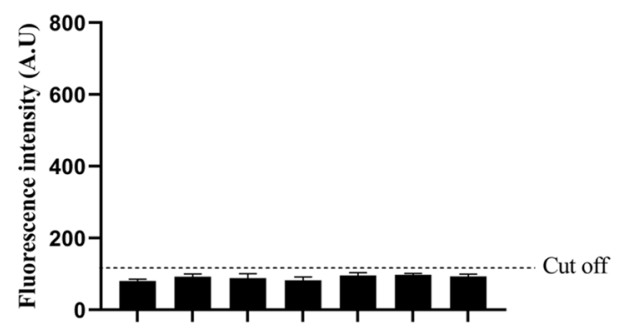

D

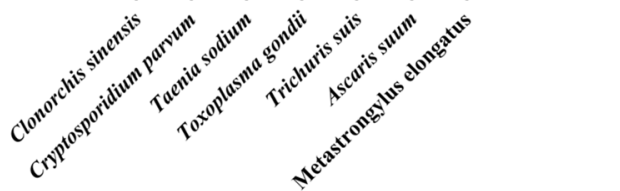

EuNPs-PAW-ES ICS

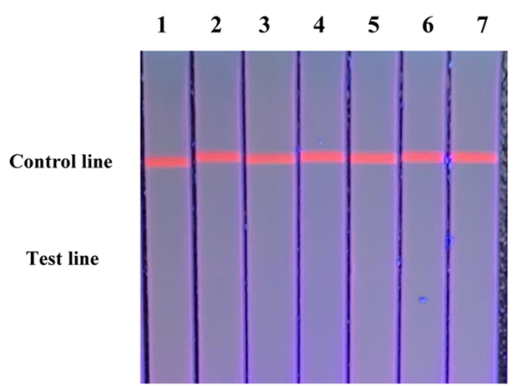

Figure 3 The specificity of ICS. In total of 38 serum samples from pigs infected with Clonorchis sinensis, Cryptosporidium parvum, Toxoplasma gondii, Taenia sodium, Ascaris suum, Trichuris suis and Meatstrongylus elongatus were detected by EuNPs-ML-ES ICS and EuNPs-PAW-ES ICS. A, C T line fluorescence values of ICS analyzed by TRF reader. B, D Visual results of ICS under ultra-violet light (1, Clonorchis sinensis 2, Cryptosporidium parvum 3, Taenia sodium 4, Toxoplasma gondii 5, Trichuris suis 6, Ascaris suum 7, Meatstrongylus elongatus).

sensitivity and specificity and has a strong agreement with standard artificial digestion in this study, which confirmed EuNPs-PAW-ES ICS has the advantages in early serodiagnosis of T. spiralis infection. Furthermore, it had been previously reported that the early antigens of T. spiralis contain early serodiagnostic markers for Trichinella infection and could be recognized by the early infection sera [15]. Our results also indicated PAW-ES antigens applied in ICS can detect $T$. spiralis infection earlier than ML-ES antigens, and it shortened effectively the window periods for detection of $T$. spiralis infection. It maybe because the the PAW-ES antigens were exposed firstly to the host's systematic immune system which triggers the generation of Trichinella-specifc antibodies during the intestinal stage of $T$. spiralis infection [35, 36].

Although colloidal gold ICS based on ML-ES antigens has been established for detection of $T$. spiralis infection $[23,24]$, fluorescent probe-based ICS as a new method was introduced in detection of T. spiralis infection, and ML-ES antigens and PAW-ES antigens were evaluated by ICS. Compared with the colloidal gold ICS, the EuNPsICS could not only distinguish the negative and positive serum by the naked eye observation, but also judge the results more precisely by the fluorescence reader. Seroconversion was firstly detected by colloidal gold ICS at 28, 21 and 18 dpi for 200, 2000 and 20000 T. spiralis ML [23], and detected by EuNPs-PAW-ES ICS at 25, 21 and $17 \mathrm{dpi}$ for 100,1000 and 10000 T. spiralis ML, which indicated EuNPs-PAW-ES ICS may be more suitable for on-site detection of T. spiralis infection.

To confirm the feasibility of method, 1032 serum samples and 1032 pork samples from the field of china were detected by ICS and artificial digestion, respectively. Interestingly, we found that three serum samples were diagnosed as antibody positive by ICS, but only one pork sample was diagnosed as positive by artificial digestion, which may be due to the low sensitivity of artificial digestion in case of light Trichinella infection [37, 38]. But, analysis of the whole results, the result of ICS is highly consistent with artificial digestion, which indicate ICS could perform detection of anti-Trichinella antibody in endemic area.

In summary, ICS based on EuNPs-ES fluorescent probe was development for a novel detection method, which allows for sensitive, rapid and low-cost detection of T. spiralis infection. EuNPs-PAW-ES ICS showed the advantages in detecting of early infection, which can be used in excluding the false negative 


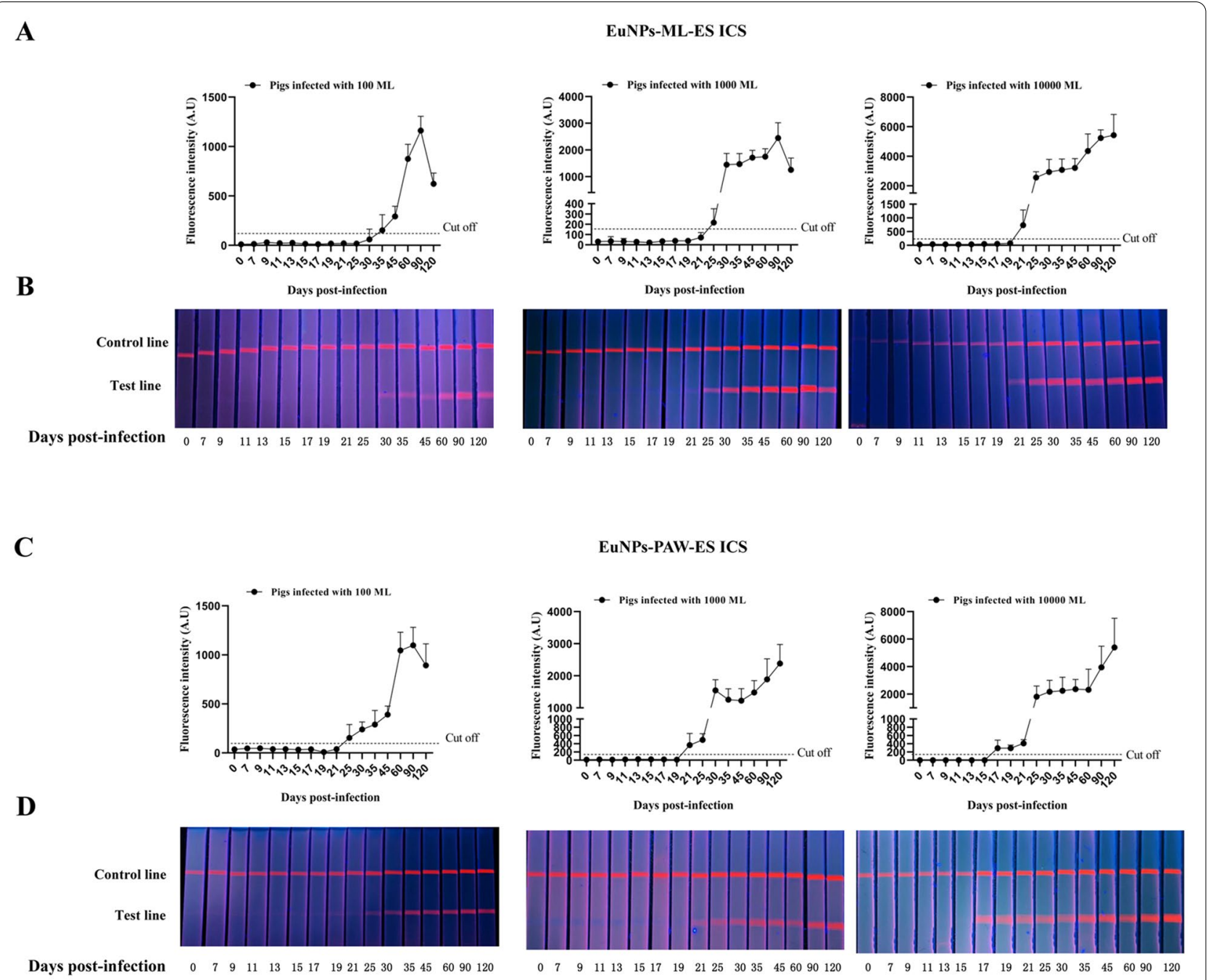

Figure 4 Seroconversion of infected pigs detected by ICS. Serum samples from pigs infected with 100, 1000 and 10 000 ML at 0,7, 9, 11, 13, 15, 17, 19, 21, 25, 30, 35, 45, 60, 90, and 120 dpi were detected by EuNPs-ML-ES ICS and EuNPs-PAW-ES ICS. A, C T-line fluorescence values analyzed by TRF reader shown as means \pm SD of five independent pigs per doses. B, D Visual results under ultra-violet light for one pig per doses.

Table 1 Seroconversion time of infected pigs detected by ICS and Qiagen ELISA

\begin{tabular}{llll}
\hline Dpi & & & \\
\hline ICS/doses $^{\mathrm{a}}$ & 100 & 1000 & 10000 \\
EuNPs-ML-ES & $30,30,35,45,45$ & $25,25,30,30,30$ & $21,21,21,21,25$ \\
EuNPs-PAW-ES & $25,25,30,30,35$ & $21,21,25,25,25$ & $17,17,19,19,19$ \\
Qiagen ELISA & $30,30,30,35,45$ & $25,25,25,30,30$ & $21,21,21,21,25$ \\
\hline
\end{tabular}

dpi: days post-infection.

doses $^{\mathrm{a}}$ : larval inoculation dose in pigs. results in early infection. These results indicated that the EuNPs-ICS were successfully established and could help inspector more efficiently screening of sera and monitoring the early T. spiralis infection. Furthermore, the reaction condition ICS need to be optimized, including the performance of blood and plasma. 
A

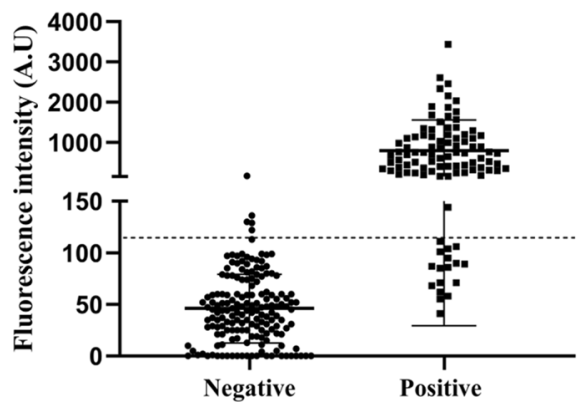

B

EuNPs-PAW-ES ICS

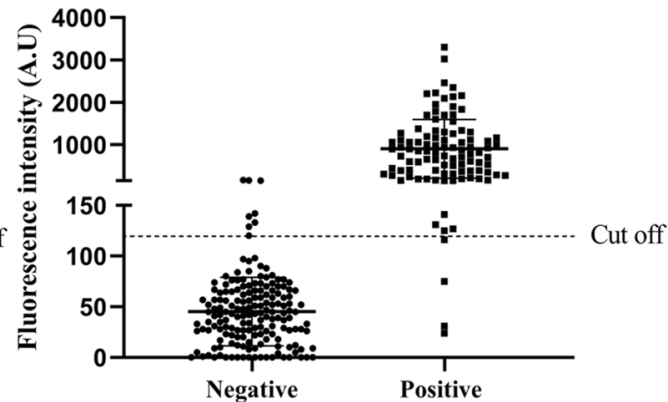

Figure 5 Distribution of results from EuNPs-ML-ES ICS and EuNPs-PAW-ES ICS. A Distribution of negative serum samples and positive serum samples detected by EuNPs-ML-ES ICS. B Distribution of negative serum samples and positive serum samples detected by EuNPs-PAW-ES ICS.

Table 2 Comparison of EuNPs-PAW-ES ICS and EuNPs-ML-ES ICS with standard artificial digestion

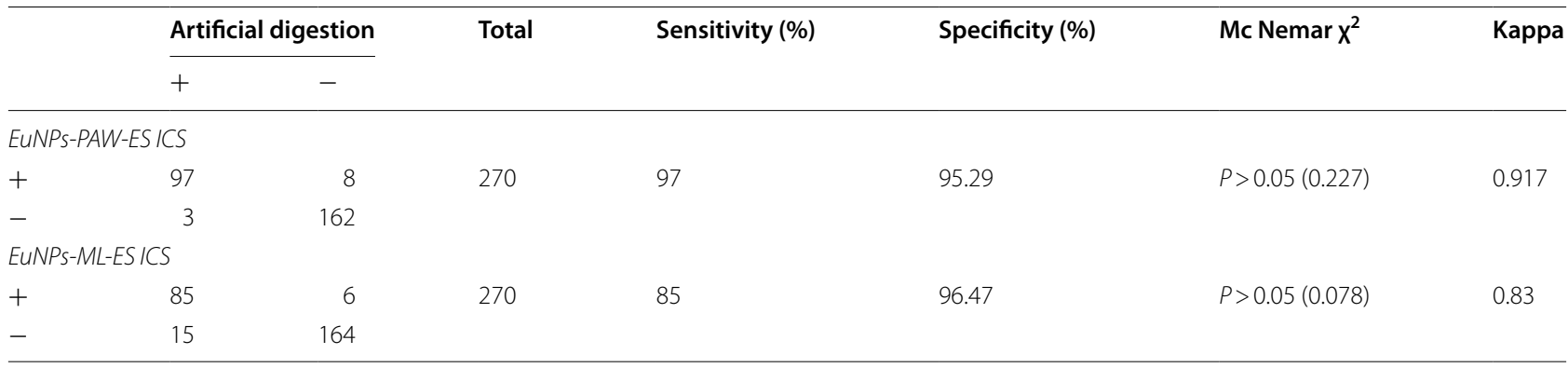

Table 3 Clinical application of ICS with artificial digestion

\begin{tabular}{llll}
\hline Method/results & Positive & Negative & In total of \\
\hline Artificial digestion & 1 & 1031 & 1032 \\
EUNPS-ML ICS & 3 & 1029 & 1032 \\
EUNPS-PAW ICS & 3 & 1029 & 1032 \\
\hline
\end{tabular}

\section{Abbreviations}

EuNPs: Eu (III) nanoparticles; ELISA: Enzyme-linked immunosorbent assay; ML: Muscle larvae; PAW: Preadult worm; dpi: Days post-infection; LPG: Larvae per gram; ICS: Immunochromatographic strip.

\section{Supplementary Information}

The online version contains supplementary material available at https://doi. org/10.1186/s13567-021-00951-9.

\section{Additional file 1. Analysis of ML-ES and PAW-ES by SDS-PAGE. A}

large number of secretion protein in the ML and PAW stage are shown in picture, respective. The results indicated that the two ES antigens can perform the next experiment.

Additional file 2. Serum samples from pigs infected with $100 \mathrm{ML}$ detected by Qiagen ELISA. (S/P values were expressed as the means \pm SD of five independent pigs).

Additional file 3. Clinical serum samples detected by ICS.
Additional file 4. Larval densities in muscles of pigs infected with different doses of $T$. spiralis at $120 \mathrm{dpi}$. By the digestion method, we took 50-100 g six parts of muscle tissues (tongue, shoulder, foreleg, diaphragm, gluteus and hind leg) from experimental pig to calculate the average lpg. This data obtained previous work in our laboratory, and had been published.

\section{Acknowledgements}

We thank Dr Xiangdong Sun and Dr Quangang Xu, China Animal Health and Epidemiology Center, Qingdao, Shandong Province, China, for helping in collecting clinical serum samples and investigating the epidemiology of Trichinella in China. Our thanks are also extended to express our gratitude to all the people who made this work.

\section{Authors' contributions}

XW: conceptualization, methodology, investigation, writing—original draft. BT: validation, formal analysis, visualization, investigation, resources. YZ: validation, visualization, investigation. JD: validation, formal analysis, investigation. NW: investigation, supervision, validation. YL: investigation, writing- review and editing. ZD: investigation, writing-review and editing. XS: investigation. QX: investigation. XL: supervision, writing-review and editing. ML: conceptualization, methodology, resources, investigation, funding. acquisition, writing-review and editing. All authors read and approved the final manuscript.

\section{Funding}

This study was supported by The National Key Research and Development Program of China (2017YFC1601200); National Natural Science Foundation of China (NSFC 31872467, 31520103916); Jilin Provincial Science and Technology Development Project (20180520042JH); and Program for JLU Science and Technology Innovative Research Team (2017TD-32). 


\section{Availability of data and materials}

All data generated or analyzed during this study are included in this published article.

\section{Declarations}

\section{Ethics statement and study samples}

The study performed the principle of the Administration of Affairs Concerning Experimental Animals in China. Experiments were given permission to the Institutional Animal Care and Use Committee of Jilin University (20170318).

\section{Competing interests}

The authors declare that they have no competing interests.

\section{Author details}

${ }^{1}$ Key Laboratory of Zoonosis Research, Ministry of Education, Institute of Zoonosis, College of Veterinary Medicine, Jilin University, Changchun 130062, China. ${ }^{2}$ Department of Nephrology, First Hospital of Jilin University, Changchun 130021, China. ${ }^{3}$ Jiangsu Co-Innovation Center for Prevention and Control of Important Animal Infectious Diseases and Zoonoses, Yangzhou, Jiangsu, China. ${ }^{4}$ College of Veterinary Medicine, College of Animal Science and Technology, Jilin Agricultural University, Changchun, China. ${ }^{5}$ China Animal Health and Epidemiology Center, Qingdao, Shandong, China.

\section{Received: 28 March 2021 Accepted: 12 May 2021}

Published online: 11 June 2021

\section{References}

1. Dupouy-Camet J (2000) Trichinellosis: a worldwide zoonosis. Vet Parasitol 93:191-200

2. Murrell KD (2016) The dynamics of Trichinella spiralis epidemiology: out to pasture? Vet Parasitol 231:92-96

3. Pozio E (2007) World distribution of Trichinella spp. infections in animals and humans. Vet Parasitol 149:3-21

4. Murrell KD, Pozio E (2011) Worldwide occurrence and impact of human trichinellosis, 1986-2009. Emerg Infect Dis 17:2194-2202

5. Bai X, Hu X, Liu X, Tang B, Liu M (2017) Current research of trichinellosis in China. Front Microbiol 8:1472

6. Cui J, Wang ZQ, Xu BL (2011) The epidemiology of human trichinellosis in China during 2004-2009. Acta Trop 118:1-5

7. Wang ZQ, Cui J, Xu BL (2006) The epidemiology of human trichinellosis in China during 2000-2003. Acta Trop 97:247-251

8. Yang XD, Xu CY, Wang SY, Gao HY, Liang JB (2020) Epidemiology, diagnosis, treatment and control measures of trichinellosis in China: an overview. Chinese J Schistosomiasis Control 32:448-452

9. Jiang $P$, Zhang $X$, Wang LA, Han LH, Yang M, Duan JY (2016) Survey of Trichinella infection from domestic pigs in the historical endemic areas of Henan province, central China. Parasitol Res 115:4707-4709

10. Gamble HR, Pozio E, Bruschi F, Nockler K, Kapel CM, Gajadhar AA (2004) International Commission on Trichinellosis: recommendations on the use of serological tests for the detection of Trichinella infection in animals and man. Parasite 11:3-13

11. Gamble HR, Rapic D, Marinculic A, Murrell KD (1988) Evaluation of excretory-secretory antigens for the serodiagnosis of swine trichinellosis. Vet Parasitol 30:131-137

12. Gondek M, Bien J, Nowakowski Z (2018) Use of ELISA and Western blot for serological detection of antibodies to E-S antigens of Trichinella spiralis muscle larvae in sera of swine experimentally infected with Trichinella spiralis. Vet Immunol Immunopathol 203:13-20

13. Wang ZQ, Shi YL, Liu RD, Jiang P, Guan YY, Chen YD (2017) New insights on serodiagnosis of trichinellosis during window period: early diagnostic antigens from Trichinella spiralis intestinal worms. Infect Dis Poverty 6:41

14. Sun GG, Wang ZQ, Liu CY, Jiang P, Liu RD, Wen H (2015) Early serodiagnosis of trichinellosis by ELISA using excretory-secretory antigens of Trichinella spiralis adult worms. Parasit Vectors 8:484
15. Yang J, Pan W, Sun X, Zhao X, Yuan G, Sun Q (2015) Immunoproteomic profile of Trichinella spiralis adult worm proteins recognized by early infection sera. Parasit Vectors 8:20

16. Liu RD, Jiang P, Wen H, Duan JY, Wang LA, Li JF (2016) Screening and characterization of early diagnostic antigens in excretory-secretory proteins from Trichinella spiralis intestinal infective larvae by immunoproteomics. Parasitol Res 115:615-622

17. Sun GG, Liu RD, Wang ZQ, Jiang P, Wang L, Liu XL (2015) New diagnostic antigens for early trichinellosis: the excretory-secretory antigens of Trichinella spiralis intestinal infective larvae. Parasitol Res 114:4637-4644

18. Bruschi F, Gomez-Morales MA, Hill DE (2019) International Commission on Trichinellosis: recommendations on the use of serological tests for the detection of Trichinella infection in animals and humans. Food Waterborne Parasitol 14:e00032

19. Gomez-Morales MA, Ludovisi A, Pezzotti P, Amati M, Cherchi S, Lalle M (2009) International ring trial to detect anti-Trichinella IgG by ELISA on pig sera. Vet Parasitol 166:241-248

20. Posthuma-Trumpie GA, Korf J, van Amerongen A (2009) Lateral flow (immuno)assay: its strengths, weaknesses, opportunities and threats. A literature survey. Anal Bioanal Chem 393:569-582

21. Chen Z, Liang R, Guo X, Liang J, Deng Q, Li M (2017) Simultaneous quantitation of cytokeratin-19 fragment and carcinoembryonic antigen in human serum via quantum dot-doped nanoparticles. Biosens Bioelectron 91:60-65

22. Guo Y, Zou R, Si F, Liang W, Zhang T, Chang Y (2020) A sensitive immunoassay based on fluorescence resonance energy transfer from up-converting nanoparticles and graphene oxide for one-step detection of imidacloprid. Food Chem 335:127609

23. Fu BQ, Li WH, Gai WY, Yao JX, Qu ZG, Xie ZZ (2013) Detection of antiTrichinella antibodies in serum of experimentally-infected swine by immunochromatographic strip. Vet Parasitol 194:125-127

24. Zhang GP, Guo JQ, Wang XN, Yang JX, Yang YY, Li QM (2006) Development and evaluation of an immunochromatographic strip for trichinellosis detection. Vet Parasitol 137:286-293

25. Xu F, Jin Z, Zou S, Chen C, Song Q, Deng S (2020) EuNPs-mAb fluorescent probe based immunochromatographic strip for rapid and sensitive detection of porcine epidemic diarrhea virus. Talanta 214:120865

26. Liang RL, Deng QT, Chen ZH, Xu XP, Zhou JW, Liang JY (2017) Europium (III) chelate microparticle-based lateral flow immunoassay strips for rapid and quantitative detection of antibody to hepatitis B core antigen. Sci Rep 7:14093

27. Wang N, Bai X, Ding J, Lin J, Zhu H, Luo X (2020) Trichinella infectivity and antibody response in experimentally infected pigs. Vet Parasitol 2020:109111

28. Bai X, Wang XL, Tang B, Shi HN, Boireau P, Rosenthal B (2016) The roles of supernatant of macrophage treated by excretory-secretory products from muscle larvae of Trichinella spiralis on the differentiation of C2C12 myoblasts. Vet Parasitol 231:83-91

29. Qiao B, Li YS, Hu P, Sun Y, Si ZZ, Lu SY (2018) EuNPs-MAb fluorescent probe based immunochromatographic strip for rapid and sensitive detection of fluorene. Sens Actuat B-Chem 262:221-227

30. Liu Y, Liu X, Li Y, Xu N, Yang Y, Liu M (2020) Evaluation of a cystatinlike protein of Trichinella spiralis for serodiagnosis and identification of immunodominant epitopes using monoclonal antibodies. Vet Parasitol 2020:109127

31. Wang Y, Hedman L, Nurmi V, Ziemele I, Perdomo MF, SoderlundVenermo M (2020) Microsphere-based IgM and IgG avidity assays for human parvovirus B19, human cytomegalovirus, and Toxoplasma gondii. mSphere 5:e00905-19

32. Willen L, Mertens P, Volf P (2018) Evaluation of the rSP03B sero-strip, a newly proposed rapid test for canine exposure to Phlebotomus perniciosus, vector of Leishmania infantum. PLoS Negl Trop Dis 12:e0006607

33. Dea-Ayuela MA, Romaris F, Ubeira FM, Rama-Iniguez S, MartinezFernandez AR, Bolas F (2001) Possible presence of common tyvelosecontaining glycans in Trichinella L1 larvae and embryonated eggs of several nematodes. Parasite 8:S120-S122

34. Yera H, Andiva S, Perret C, Limonne D, Boireau P, Dupouy-Camet J (2003) Development and evaluation of a Western blot kit for diagnosis of human trichinellosis. Clin Diagn Lab Immunol 10:793-796 
35. Wang ZQ, Liu RD, Sun GG, Song YY, Jiang P, Zhang X (2017) Proteomic analysis of Trichinella spiralis adult worm excretory-secretory proteins recognized by sera of patients with early trichinellosis. Front Microbiol 8:986

36. Liu RD, Qi X, Sun GG, Jiang P, Zhang X, Wang LA (2016) Proteomic analysis of Trichinella spiralis adult worm excretory-secretory proteins recognized by early infection sera. Vet Parasitol 231:43-46

37. Gamble HR (1998) Sensitivity of artificial digestion and enzyme immunoassay methods of inspection for trichinae in pigs. J Food Prot 61:339-343
38. Forbes LB, Gajadhar AA (1999) A validated Trichinella digestion assay and an associated sampling and quality assurance system for use in testing pork and horse meat. J Food Prot 62:1308-1313

\section{Publisher's Note}

Springer Nature remains neutral with regard to jurisdictional claims in published maps and institutional affiliations. 